\title{
Entanglement of a Mesoscopic Field with an Atom induced by Photon Graininess in a Cavity
}

\author{
A. Auffeves,${ }^{1}$ P. Maioli, ${ }^{1}$ T. Meunier,${ }^{1}$ S. Gleyzes,${ }^{1}$ G. \\ Nogues, ${ }^{1}$ M. Brune, ${ }^{1}$ J.M. Raimond,${ }^{1}$ and S. Haroche ${ }^{1,2}$ \\ ${ }^{1}$ Laboratoire Kastler Brossel, Département de Physique de l'Ecole Normale Supérieure, \\ 24 rue Lhomond, F-75231 Paris Cedex 05, France \\ ${ }^{2}$ Collège de France, 11 place Marcelin Berthelot, F-75231 Paris Cedex 05, France
}

(Dated: November 3, 2018)

\begin{abstract}
We observe that a mesoscopic field made of several tens of microwave photons exhibits quantum features when interacting with a single Rydberg atom in a high- $Q$ cavity. The field is split into two components whose phases differ by an angle inversely proportional to the square root of the average photon number. The field and the atomic dipole are phase-entangled. These manifestations of photon graininess vanish at the classical limit. This experiment opens the way to studies of large Schrödinger cat states at the quantum-classical boundary.
\end{abstract}

PACS numbers: 03.65.-w, 03.67.Mn, 42.50.Dv 
Known since the early days of molecular beams, the Rabi oscillation of a two-level atom in a coherent field plays a fundamental role in quantum optics. When the field is classical (i.e. made of a huge number of photons whose graininess is negligible) it is not affected by the coupling to the atom, which oscillates between the two levels at a frequency proportional to the field amplitude. When the field contains no photons, a situation relevant to the Cavity Quantum Electrodynamics (CQED) context [1], the Rabi oscillation occurs at the much slower vacuum Rabi frequency $\Omega$, proportional to the zero-point field fluctuations in the cavity. The field, oscillating between the 0 and 1 photon states, is then strongly affected by the coupling. The Rabi oscillation results in periodic maximum atom-field entanglement [2] and in other various atom-field effects either in the optical [3, 4, 5] or microwave [6] domains.

In the intermediate regime, for mesoscopic fields with an average photon number $\bar{n} \sim$ a few tens, the amplitude of the Rabi oscillation is predicted to collapse and revive periodically [7]. The collapse is usually attributed to the fluctuation of the field amplitude, and the revival to the graininess of the photon number. Entanglement between the atom and the field plays also an important role in the process [8, 9, 10, 11]. Due to the spread of the Rabi frequencies corresponding to different photon numbers, the atom gets entangled with the field in a quantum superposition of two coherent components rotating in opposite directions in phase space. These components are correlated to two different atomic state superpositions. The Rabi oscillation appears as a quantum interference effect between the amplitudes associated to these atomic superpositions. Its collapse is a direct consequence of complementarity. When the field is split into two orthogonal components with different phases, it stores an unambiguous information about these atomic states, thus destroying the interference between them. The Rabi oscillation revives when this information is erased, as the two components of the field periodically merge together.

Evidence of the collapse and revival of the Rabi oscillation has been obtained in CQED experiments [12, 13] with fields containing about one photon. Collapses and revivals have also been observed in ion-traps with $\sim 3$ ion vibration quanta replacing the photons [14]. These experiments focused on the atomic evolution in a regime where only a few photons/phonons were involved.

We describe here an experiment detecting the evolution of a mesoscopic field containing an average photon number of up to about 40 and interacting with a single atom in a high $Q$ microwave cavity. The phase splitting of the field into two components rotating in opposite 
directions in phase space is observed by a homodyne method [15, 16]. A single atom splits the field into phase components separated by up to 90 degrees, entangled with atomic states having initially opposite expectation values of their dipole. This experiment demonstrates that an atom leaves its imprint on a mesoscopic field, opening the way to applications in CQED.

A two-level atom $(e, g)$, initially in $g$, and a single resonant field mode containing $n+1$ photons at $t=0$, evolve into the state at time $t\left|\psi_{n}(t)\right\rangle=\cos (\Omega \sqrt{n+1} t / 2)$ $|g, n+1\rangle-i \sin (\Omega \sqrt{n+1} t / 2)|e, n\rangle$. This describes a reversible oscillation between an atom in $e$ with $n$ photons and in $g$ with $n+1$ photons. We are interested here, rather, in the situation where the field is initially in a coherent state, superposition of number states $|n\rangle$, defined as $|\alpha\rangle=e^{-|\alpha|^{2} / 2} \sum_{n}\left(\alpha^{n} / \sqrt{n !}\right)|n\rangle$ (where $\alpha$ is taken real without loss of generality). The mean photon number is $\bar{n}=|\alpha|^{2}$. The photon number and phase fluctuations are $\Delta n=\sqrt{\bar{n}}=|\alpha|$ and $\Delta \phi=1 / \Delta n=1 / \sqrt{\bar{n}}$ respectively. The atom-field state at time $t$ can be derived exactly by superposition of the partial states $\left|\psi_{n}(t)\right\rangle$ associated to the $|n\rangle$ states in the expansion of $|\alpha\rangle$. This solution describes a beating between probabilities evolving at the incommensurate frequencies $\Omega \sqrt{n+1}$.

A more transparent expression for the system's state is obtained for large $\bar{n}$, where the relative fluctuation of the photon numbers is small [8, 9, 10, 11]. It is then legitimate to replace in the exact expression $\sqrt{n+1}$ by $\sqrt{n}+1 /(2 \sqrt{\bar{n}})$ and to develop then all functions of $n$ around $\bar{n}$ up to second order in $n-\bar{n}$. The system's state in the interaction picture then becomes at time $t$ :

$$
\begin{aligned}
|\psi(t)\rangle \simeq & \frac{1}{\sqrt{2}}\left[e^{-i \Omega \sqrt{\bar{n}} t / 2}\left|\alpha^{+}(t)\right\rangle\left|\phi_{a}^{+}(t)\right\rangle\right. \\
& \left.-e^{i \Omega \sqrt{\bar{n}} t / 2}\left|\alpha^{-}(t)\right\rangle\left|\phi_{a}^{-}(t)\right\rangle\right]
\end{aligned}
$$

where the field states $\left|\alpha^{ \pm}(t)\right\rangle$ and atomic states $\left|\phi_{a}^{ \pm}(t)\right\rangle$ are:

$$
\begin{gathered}
\left|\alpha^{ \pm}(t)\right\rangle=e^{-|\alpha|^{2} / 2} e^{ \pm i \Omega \sqrt{\bar{n}} t / 4} \sum_{n} e^{ \pm i \Omega(n-\bar{n})^{2} t /\left(16 \bar{n}^{3 / 2}\right)} \times \\
\times \frac{\left(\alpha e^{\mp i \Omega t / 4 \sqrt{\bar{n}}}\right)^{n}}{\sqrt{n !}}|n\rangle \\
\left|\phi_{a}^{ \pm}(t)\right\rangle=\frac{1}{\sqrt{2}}\left[e^{\mp i \Omega t / 4 \sqrt{\bar{n}}}|e\rangle \pm|g\rangle\right] .
\end{gathered}
$$

From Eq.(1), we derive the probability $P_{g}(t)$ to detect at time $t$ the atom in level $g$ :

$$
P_{g}(t)=\frac{1}{2}\left[1+\Re\left(e^{-i \Omega \sqrt{\bar{n}} t}\left\langle\alpha^{-}(t) \mid \alpha^{+}(t)\right\rangle\right)\right]
$$


Eqs.(14) yield a synthetic view of the evolution of a mesoscopic field coupled to a single atom. At time $t=0$, Eqs. (21) and (3) reduce to $\left|\alpha^{ \pm}(0)\right\rangle=|\alpha\rangle$ and $\left|\phi_{a}^{ \pm}(0)\right\rangle=(|e\rangle \pm|g\rangle) / \sqrt{2}$. The initial atomic state $|g\rangle$ appears as the superposition of two orthogonal "dipole states" with a non-zero mean dipole either in phase $[(|e\rangle+|g\rangle) / \sqrt{2}]$ or $\pi$-out of phase $[(|e\rangle-|g\rangle) / \sqrt{2}]$ with the field. As time proceeds, the phases of these two dipole states rotate in opposite directions in phase space at frequency $\pm \Omega /(4 \sqrt{\bar{n}})$ [Eq.(3)]. Simultaneously, the field splits into two quasi-coherent components with phases $\Phi^{ \pm}= \pm \Omega t / 4 \sqrt{\bar{n}}$, each remaining locked in phase or $\pi$-out of phase with the two rotating atomic "dipole states" [Eqs.(11) and (2)]. The field components have their phases not only shifted, but also spread out. The amplitude factor $\left[\alpha \exp (\mp i \Omega t /(4 \sqrt{\bar{n}})]^{n}\right.$ in Eq.(2) accounts for the phase drift, while the factor whose exponent is quadratic in $n$ is responsible for phase spreading.

As a result of the atom-field evolution [Eq.(10)], the two systems are generally entangled. The probability amplitudes associated with the two components of this entangled state evolve at frequency $\pm \Omega \sqrt{\bar{n}} / 2,2 \bar{n}$ times larger than the drift frequency of the atomic and field phases. The beating between these two fast oscillating amplitudes leads to the Rabi oscillation [Eq.(44)], which appears as an atomic interference effect. The envelope of the Rabi oscillation is the overlap between the two field components, a clear manifestation of complementarity. Note that this discussion is also valid, within minor changes, when the atom is initially in $e$.

The mesoscopic nature of the field is essential. If the photon number is microscopic $(\bar{n} \leq$ 10), the approximations leading to Eqs.(14) are not valid. The classical limit corresponds conversely to $\Omega \rightarrow 0, \bar{n} \rightarrow \infty$, with $\Omega \sqrt{\bar{n}}$ constant. The graininess of the photon number is then washed out, with collapse and revival times pushed out to infinity.

Our CQED set-up, sketched in Fig.1(a), is described in detail in [2]. ${ }^{85} \mathrm{Rb}$ atoms, effusing from oven $O$, are velocity-selected by laser optical pumping and prepared in zone $B$ in the circular Rydberg state with principal quantum number 51 (level $e$ ) or 50 (level $g$ ) by a combination of laser and radiofrequency excitations. The atomic preparation is pulsed, so that the position of each atom is known along the beam within $\pm 1 \mathrm{~mm}$. The atoms cross, one at a time, the cavity $C$ sustaining a Gaussian field mode (waist $w=6 \mathrm{~mm}$ ) exactly resonant with the $e \rightarrow g$ transition at $51.1 \mathrm{GHz}$. The cavity, cooled to $0.6 \mathrm{~K}$, is made up of two superconducting niobium mirrors. The vacuum Rabi frequency is $\Omega=3.10^{5} \mathrm{~s}^{-1}$. The atom and field relaxation times are $T_{a}=30 \mathrm{~ms}$ and $T_{c a v}=850 \mu \mathrm{s}$, corresponding to 
$\Omega T_{\text {cav }}=250$, fulfilling the strong coupling regime condition. The atomic velocity is chosen from two values, $v_{a}=335 \mathrm{~m} / \mathrm{s}$ and $v_{b}=200 \mathrm{~m} / \mathrm{s}$, corresponding to an effective atom-cavity interaction time $t_{i}=t_{a}=\sqrt{\pi} w / v_{a}=32 \mu \mathrm{s}$ or $t_{i}=t_{b}=\sqrt{\pi} w / v_{b}=53 \mu \mathrm{s}$. The atoms are detected after $C$ by a field-ionization detector $D$ (quantum efficiency $\sim 70 \%$ ) discriminating $e$ and $g$.

A coherent field, produced by a pulsed (duration $23 \mu \mathrm{s}$ ) microwave source $S$, is injected in $C$ through a small hole in a mirror. The average photon number $\bar{n}$ is controlled with attenuators. An independent measurement of $\bar{n}$ (with a precision of $\pm 10 \%$ ) is realized by detecting the light shift produced by the field on a Rydberg atom [17].

The timing of the experimental sequence is shown on the space-time diagram of Fig. 1(b). The preparation box $B$, cavity $C$ and detector $D$ are represented, from left to right, by vertical grey bands. The cavity is initially in the vacuum state. A first atom $A_{1}$, prepared in level $e$ or $g$ with velocity $v_{a}$ or $v_{b}$ is sent across the set-up (lower diagonal line). Just before $A_{1}$ reaches $C$, a coherent field $F_{1}$ is injected into the mode (lower horizontal line). The atom then crosses $C$. As soon as it exits the mode, a probe field $F_{2}$ (upper horizontal line) is injected, with the same amplitude as $F_{1}$, and a relative phase $\phi+\pi$. The $F_{1}$ and $F_{2}$ fields add in $C$ and their sum is read out by a second, probe atom $A_{2}$ prepared in $g$, reaching $C$ just after the injection of $F_{2}$ (upper diagonal line).

This procedure amounts to a homodyne phase sensitive detection [15, 16]. Atom $A_{2}$ absorbs the final field in $C$, ending up in $e$ with a large probability when $\phi$ is such that there is one photon or more in $C$. By repeating the sequence many times, we construct a signal $S_{g}(\phi)$ equal to the probability versus $\phi$ that $A_{2}$ remains in $g$. This signal exhibits peaks revealing the final phase pattern of the field in $C$ after interaction with $A_{1}$. The $A_{2}$ signal is observed in coincidence with the detection of $A_{1}$.

Fig. 2 shows $S_{g}(\phi)$ for the two $A_{1}-F_{1}$ interaction times $t_{a}[2(\mathrm{a})]$ and $t_{b}[2(\mathrm{~b})]$. The signals are plotted versus $\phi$ for different mean photon numbers $\bar{n}$ in the range $\bar{n}=15$ to 36 . Fig. 2(b) also shows the signal without $A_{1}$, for $\bar{n}=29$ photons, whose peak is centered around phase origin $\phi=0$. The splitting by a single atom of the field into two symmetrical components with different phases is clearly visible in the other recordings. For a given interaction time, the splitting decreases with field amplitude and, for a given $\bar{n}$, the separation increases with time. Fig. 2(c) summarizes the results by plotting the phases of the two components as a function of the dimensionless parameter $\Phi^{+}=\Omega t / 4 \sqrt{\bar{n}}$. The 
dotted line corresponds to the phases $\Phi^{ \pm}$predicted by Eq.(11). The solid line results from a numerical simulation solving the exact field equation of motion and taking into account cavity damping. The agreement between the experimental points and the solid lines is very good. The maximum phase splitting observed, for $\bar{n}=15$ photons and $t_{i}=52 \mu$ s, is 90 degrees.

The inset in Fig. 2(c) shows, for $\bar{n}=36$ and $t_{i}=t_{a}$ the Wigner function $W\left(\beta_{x}+i \beta_{y}\right)$ of the field in the cavity. It results from the explicit numerical simulation. The field state is computed for an atom found in $g, 48 \mu$ s after its crossing of the cavity axis. This $W$ function clearly exhibits the two separate field components and interferences which are a signature of a "Schrödinger cat" coherence. The square of the distance in phase space between the two components, $d^{2}=4 \bar{n} \sin ^{2}(\Omega t / 4 \sqrt{\bar{n}})$, is a measure of the mesoscopic character of this superposition. In the range of $\bar{n}$ we have explored, $d^{2}$ is nearly constant versus $\bar{n}$, equal to $\sim 20$ for $t_{i}=t_{a}$ and $\sim 40$ for $t_{i}=t_{b}$. These figures are to be compared with the size of the Schrödinger cats realized in our earlier CQED experiment $\left(d^{2} \leq 8\right)$ [18]. Larger "cat" states are obtained here, taking advantage of a higher- $Q$ cavity and of the resonant atom-field interaction, which achieves a larger phase splitting than the dispersive coupling used in [18]. The theoretical decoherence time of the final cat state, $2 T_{\text {cav }} / d^{2}$, is $\sim 43 \mu$ s for $t_{i}=t_{b}$, meaning that the superposition loses its coherence as fast as it is generated. The situation is better for the smaller "cats" prepared faster $\left(t_{i}=t_{a}\right)$, which have a decoherence time $\sim 85 \mu \mathrm{s}$.

We have also checked the correlation between the atomic state and the field phase by selectively preparing $\left|\phi_{a}^{+}(0)\right\rangle$ or $\left|\phi_{a}^{-}(0)\right\rangle$ at the beginning of the interaction, within a time short enough so that the slow phase drift of the atom and field states can be neglected. To prepare $\left|\phi_{a}^{+}(0)\right\rangle$, the atom, initially in $g$, first performs a $\pi / 2$ Rabi pulse according to the transformation $g \rightarrow\left[e^{-i \pi / 4}\left|\phi_{a}^{+}(0)\right\rangle-e^{i \pi / 4}\left|\phi_{a}^{-}(0)\right\rangle\right] / \sqrt{2}$. The atom is then detuned by Stark effect with respect to the cavity, during a time much shorter than the Rabi period. A pulse of electric field is applied between the cavity mirrors, whose effect is to shift by $\pi / 2$ the relative phase of the $e$ and $g$ states [2]. The sequence of Rabi and Stark pulses transforms the initial $g$ state, superposition of the interfering $\left|\phi_{a}^{+}(0)\right\rangle$ and $\left|\phi_{a}^{-}(0)\right\rangle$ states, into $\left|\phi_{a}^{+}(0)\right\rangle$ alone. The system ends up in the slowly evolving quasi-stationary state described by the first term in the right hand side of Eq.(11). The atom and the field subsequently drift in phase in only one direction. We observe that the Rabi oscillation is frozen from then on. 
A homodyne measurement of the field phase after the atom exits from $C$ then reveals, as expected, only a single phase-shifted field component (open circles in Fig. 3). Similarly, we have prepared $\left|\phi_{a}^{-}(0)\right\rangle$ by applying the same Rabi and Stark switching pulse sequence starting from level $e$. This state couples to the other component of the field as revealed by the subsequent homodyne detection (solid squares in Fig. 3). This experiment clearly demonstrates correlations between the atomic state and the field phase.

Further tests of the quantum coherence in this system are under way. After collapse of the Rabi oscillation, we apply to the atom, at a time $T$, a Stark pulse switching the signs of the quantum amplitudes associated to $e$ and $g$. According to Eq.(II), this pulse suddenly exchanges the atomic states correlated to the $\left|\alpha^{+}(t)\right\rangle$ and $\left|\alpha^{-}(t)\right\rangle$ field components. The atom-field coupling resumes afterwards, reversing the sign of rotation of the two field components. At time $2 T$, the two field states are back in phase and the Rabi oscillation revives, revealing the coherent nature of the atom/cavity state. This induced revival, similar to a spin-echo, will be described in a forthcoming paper.

We have shown that a single atom leaves a quantum imprint on a mesoscopic field made of several tens of photons. This experiment illustrates a striking feature of quantum-classical correspondence. The $1 / \sqrt{\bar{n}}$ dependence of $\Phi^{+}$shows that the bigger the mesoscopic system is, the longer is the time required for the atom to pass its "quantumness" to the field. At the classical limit this time goes to infinity. Using better cavities and slower atoms should allow us to prepare mesoscopic state superpositions with $\bar{n}$ in the range of a few hundred. We plan to study the properties of these states and their decoherence. At a practical level, the large phase shifts produced by a single atom on a mesoscopic field could be used to develop new atomic detection schemes. The information carried by a single atom can be transferred to a field containing a large number of photons, which can be subsequently read out by a sample made of many probe atoms. This amplifying effect opens promising perspectives for $\mathrm{a} \sim 100 \%$ atomic detection efficiency in microwave CQED experiments.

Laboratoire Kastler Brossel is a laboratory of Université Pierre et Marie Curie and ENS, associated to CNRS (UMR 8552). We acknowledge support of the European Community, of the Japan Science and Technology corporation (International Cooperative Research Project : Quantum Entanglement). 
[1] H. Mabuchi and A. C. Doherty, Science 298, 1372 (2002).

[2] J.-M. Raimond, M. Brune, and S. Haroche, Rev. Mod. Phys. 73, 565 (2001).

[3] S. L. Mielke, G. T. Foster, and L. A. Orozco, Phys. Rev. Lett. 80, 3948 (1998).

[4] C. J. Hood, T. W. Lynn, A. C. Doherty, A. S. Papkins, and H. J. Kimble, Science 287, 1447 (2000).

[5] T. Fischer, P. Maunz, P. W. H. Pinkse, T. Puppe, and G. Rempe, Phys. Rev. Lett. 88, 163002 (2002).

[6] M. Weidinger, B. Varcoe, R. Heerlein, and H. Walther, Phys. Rev. Lett. 82, 3795 (1999).

[7] J. Eberly, N. Narozhny, and J. Sanchez-Mondragon, Phys. Rev. Lett. 44, 1323 (1980).

[8] J. Gea-Banacloche, Phys. Rev. Lett. 65, 3385 (1990).

[9] J. Gea-Banacloche, Phys. Rev. A 44, 5913 (1991).

[10] V. Buzek, H. Moya-Cessa, P. Knight, and S. Phoenix, Phys. Rev. A 45, 8190 (1992).

[11] V. Buzek and P. Knight, in Progress in Optics XXXIV (Elsevier, 1995), vol. 34, p. 1.

[12] G. Rempe, H. Walther, and N. Klein, Phys. Rev. Lett. 58, 353 (1987).

[13] M. Brune, F. Schmidt-Kaler, A. Maali, J. Dreyer, E. Hagley, J.-M. Raimond, and S. Haroche, Phys. Rev. Lett. 76, 1800 (1996).

[14] D. M. Meekhof, C. Monroe, B. E. King, W. M. Itano, and D. J. Wineland, Phys. Rev. Lett. 76, 1796 (1996).

[15] A. Rauschenbeutel, G. Nogues, S. Osnaghi, P. Bertet, M. Brune, J.-M. Raimond, and S. Haroche, Phys. Rev. Lett. 83, 5166 (1999).

[16] P. Bertet, A. Auffeves, P. Maioli, S. Osnaghi, T. Meunier, M. Brune, J.-M. Raimond, and S. Haroche, Phys. Rev. Lett. 89, 200402 (2002).

[17] M. Brune, P. Nussenzveig, F. Schmidt-Kaler, F. Bernardot, A. Maali, J.-M. Raimond, and S. Haroche, Phys. Rev. Lett. 72, 3339 (1994).

[18] M. Brune, E. Hagley, J. Dreyer, X. Maître, A. Maali, C. Wunderlich, J.-M. Raimond, and S. Haroche, Phys. Rev. Lett. 77, 4887 (1996). 


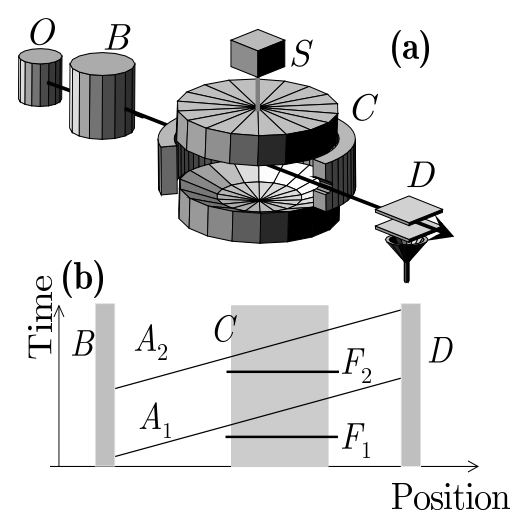

FIG. 1: (a) Experimental apparatus. (b) Timing of the experiment.

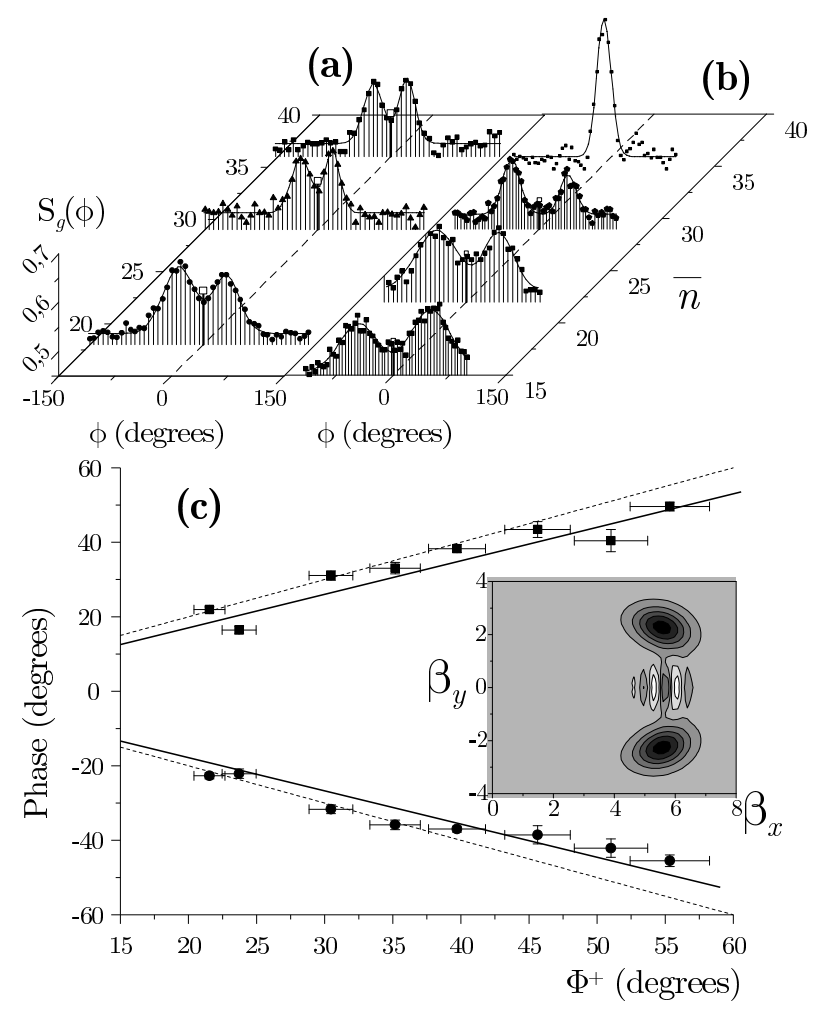

FIG. 2: (a) Field phase distribution $S_{g}(\phi)$ for three $\bar{n}$ values $(18,29$ and 36$)$ and $t_{i}=t_{a}=32 \mu$ s. The points are experimental and the curves fits on a sum of Gaussians. The thick vertical line indicates phase reference. (b) Field phase distribution for $\bar{n}=15,22$ and 29 and $t_{i}=t_{b}=53 \mu$ s. Upper curve: phase reference (no atom $A_{1}$ and $\bar{n}=29$; for clarity, this curve has been translated along the $\bar{n}$ axis). (c) Phases of the two field components versus $\Phi^{+}=\Omega t / 4 \sqrt{\bar{n}}$. Dotted and solid lines are theoretical (see text). Inset: computed cavity field Wigner function $W\left(\beta_{x}+i \beta_{y}\right)$ for $\bar{n}=36$ and $t_{i}=t_{a}$. 


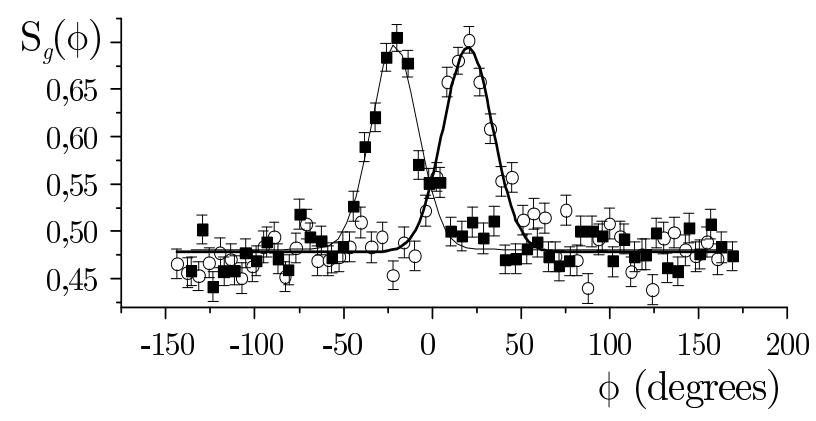

FIG. 3: Field phase distributions $S_{g}(\phi)$ following preparation of atomic states $\left|\phi_{a}^{ \pm}(0)\right\rangle$ by combination of Rabi and Stark pulses $\left(\bar{n}=27\right.$ and $\left.t_{i}=t_{a}\right)$. Open circles: preparation of $\left|\phi_{a}^{+}(0)\right\rangle$. Solid squares: preparation of $\left|\phi_{a}^{-}(0)\right\rangle$. Solid lines are gaussian fits. 